\title{
High-quality graphene flakes exfoliated on a flat hydrophobic polymer
}

Pedrinazzi, Paolo; Caridad, Jose M.; Mackenzie, David M. A.; Pizzocchero, Filippo; Gammelgaard, Lene; Jessen, Bjarke S.; Sordan, Roman; Booth, Timothy J.; Bøggild, Peter

Published in:

Applied Physics Letters

Link to article, DOI:

$10.1063 / 1.5009168$

Publication date:

2018

Document Version

Publisher's PDF, also known as Version of record

Link back to DTU Orbit

Citation (APA):

Pedrinazzi, P., Caridad, J. M., Mackenzie, D. M. A., Pizzocchero, F., Gammelgaard, L., Jessen, B. S., Sordan, R., Booth, T. J., \& Bøggild, P. (2018). High-quality graphene flakes exfoliated on a flat hydrophobic polymer. Applied Physics Letters, 112(3), [033101 ]. https://doi.org/10.1063/1.5009168

\section{General rights}

Copyright and moral rights for the publications made accessible in the public portal are retained by the authors and/or other copyright owners and it is a condition of accessing publications that users recognise and abide by the legal requirements associated with these rights.

- Users may download and print one copy of any publication from the public portal for the purpose of private study or research.

- You may not further distribute the material or use it for any profit-making activity or commercial gain

- You may freely distribute the URL identifying the publication in the public portal 


\section{High-quality graphene flakes exfoliated on a flat hydrophobic polymer}

Paolo Pedrinazzi, José M. Caridad, David M. A. Mackenzie, Filippo Pizzocchero, Lene Gammelgaard, Bjarke S. Jessen, Roman Sordan, Timothy J. Booth, and Peter Bøggild

Citation: Appl. Phys. Lett. 112, 033101 (2018);

View online: https://doi.org/10.1063/1.5009168

View Table of Contents: http://aip.scitation.org/toc/apl/112/3

Published by the American Institute of Physics

\section{Articles you may be interested in}

Manipulation of electron transport in graphene by nanopatterned electrostatic potential on an electret Applied Physics Letters 112, 033102 (2018); 10.1063/1.5006226

Topological optical isolator based on polariton graphene

Applied Physics Letters 112, 031106 (2018); 10.1063/1.5018902

The interplay between excitons and trions in a monolayer of $\mathrm{MoSe}_{2}$

Applied Physics Letters 112, 031107 (2018); 10.1063/1.5019177

Broadband nonlinear optical response of monolayer $\mathrm{MoSe}_{2}$ under ultrafast excitation Applied Physics Letters 112, 031108 (2018); 10.1063/1.5010060

Modification of graphene electronic properties via controllable gas-phase doping with copper chloride Applied Physics Letters 112, 033107 (2018); 10.1063/1.5006001

High breakdown electric field in $\beta-\mathrm{Ga}_{2} \mathrm{O}_{3} /$ graphene vertical barristor heterostructure

Applied Physics Letters 112, 032101 (2018); 10.1063/1.5002138

\section{Scilight}

Sharp, quick summaries illuminating the latest physics research

\section{Sign up for FREE!}

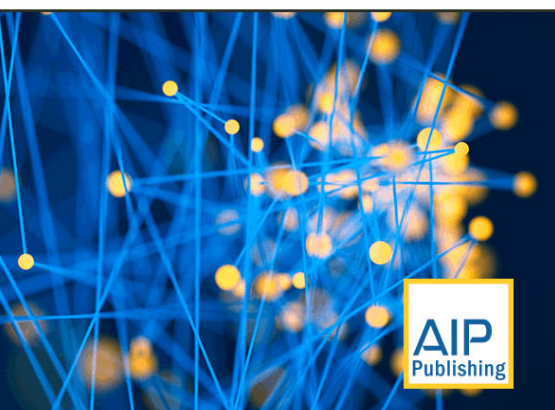




\title{
High-quality graphene flakes exfoliated on a flat hydrophobic polymer
}

\author{
Paolo Pedrinazzi, ${ }^{1,2, a)}$ José M. Caridad, ${ }^{1, a), b)}$ David M. A. Mackenzie, ${ }^{1}$ Filippo Pizzocchero, ${ }^{1}$ \\ Lene Gammelgaard, ${ }^{1}$ Bjarke S. Jessen, ${ }^{1}$ Roman Sordan, ${ }^{2}$ Timothy J. Booth, ${ }^{1}$ \\ and Peter Bøggild ${ }^{1, b)}$ \\ ${ }^{1}$ Department of Micro- and Nanotechnology, Centre for Nanostructured Graphene (CNG), \\ Technical University of Denmark, 2800 Kongens Lyngby, Denmark \\ ${ }^{2}$ L-NESS Laboratory, Department of Physics, Politecnico di Milano, Via Anzani 42, 22100 Como, Italy
}

(Received 13 October 2017; accepted 29 December 2017; published online 16 January 2018)

\begin{abstract}
We show that graphene supported on a hydrophobic and flat polymer surface results in flakes with extremely low doping and strain as assessed by their Raman spectroscopic characteristics. We exemplify this technique by micromechanical exfoliation of graphene on flat poly(methylmethacrylate) layers and demonstrate Raman peak intensity ratios $\mathrm{I}(2 \mathrm{D}) / \mathrm{I}(\mathrm{G})$ approaching 10 , similar to pristine freestanding graphene. We verify that these features are not an artifact of optical interference effects occurring at the substrate: they are similarly observed when varying the substrate thickness and are maintained when the environment of the graphene flake is completely changed, by encapsulating preselected flakes between hexagonal boron nitride layers. The exfoliation of clean, pristine graphene layers directly on flat polymer substrates enables high performance, supported, and non-encapsulated graphene devices for flexible and transparent optoelectronic studies. We additionally show that the access to a clean and supported graphene source leads to high-quality van der Waals heterostructures and devices with reproducible carrier mobilities exceeding $50000 \mathrm{~cm}^{2} \mathrm{~V}^{-1} \mathrm{~s}^{-1}$ at room temperature. Published by AIP Publishing. https://doi.org/10.1063/1.5009168
\end{abstract}

Graphene is typically prepared on oxidized highly doped silicon substrates, which enables the reliable exfoliation of flakes, ${ }^{1}$ transfer of wafer-scale films, ${ }^{2}$ and the optical identification of monolayers due to significant contrast enhancement for selected oxide thicknesses ${ }^{3}$ and allows its electrical characterization. ${ }^{4,5}$ Despite the possibility of maximizing yields and size of flakes by oxygen plasma pre-treatments on oxidized silicon, ${ }^{1}$ the direct contact with the $\mathrm{SiO}_{2}$ layer can adversely affect the performance of graphene devices in several ways. First, the corrugation of graphene on $\mathrm{SiO}_{2}$ induces the bending of $\mathrm{sp}^{2}$ bonds in the basal plane of the monolayer, which is believed to lower the energy barrier for water and oxygen adsorption. ${ }^{6,7}$ Also, residual charged impurities arising from charge traps inside $\mathrm{SiO}_{2}$, or water molecules adsorbed on the surface, can lead to additional unwanted doping and carrier scattering in graphene devices. ${ }^{6,7}$ Even in the absence of adsorbed molecules, polar optical phonons in $\mathrm{SiO}_{2}$ impose an upper limit on the room temperature carrier mobility of around $40000 \mathrm{~cm}^{2} \mathrm{~V}^{-1} \mathrm{~s}^{-1,}$, far away from intrinsic limits predicted for monolayer graphene of $\sim 120000 \mathrm{~cm}^{2} \mathrm{~V}^{-1} \mathrm{~s}^{-1}$. In particular, typical graphene on $\mathrm{SiO}_{2}$ devices exhibits a carrier mobility below $5000 \mathrm{~cm}^{2} \mathrm{~V}^{-1} \mathrm{~s}^{-1,4,5,10}$ due to combinations of several of these effects. Raman spectroscopic measurements can be used to predict the impact of these effects on electronic properties prior to device fabrication: for graphene on $\mathrm{SiO}_{2}$, a low intensity ratio of the 2D peak and $\mathrm{G}$ peak $\mathrm{I}(2 \mathrm{D}) / \mathrm{I}(\mathrm{G}) \sim 1$ is observed, whereas pristine, suspended graphene shows $\mathrm{I}(2 \mathrm{D}) / \mathrm{I}(\mathrm{G})$ ratios up to 10 (Ref. 11).

Strategies already exist for mitigating these detrimental factors, including transferring graphene onto hexagonal boron

\footnotetext{
${ }^{a)}$ P. Pedrinazzi and J. M. Caridad contributed equally to this work.

b) Authors to whom correspondence should be addressed: jcar@nanotech. dtu.dk and peter.boggild@nanotech.dtu.dk
}

nitride $(\mathrm{hBN})$ as a substrate to reduce the charged traps in the substrate, graphene corrugation and substrate-phonon scattering, ${ }^{12}$ or rendering the oxide surface hydrophobic through the use of self-assembled monolayers (SAMs) to avoid water contaminants. ${ }^{13-15}$ All these techniques result in increased electrical and optical performance, with measured carrier mobilities from 10000 to $40000 \mathrm{~cm}^{2} \mathrm{~V}^{-1} \mathrm{~s}^{-1}$ and $\mathrm{I}(2 \mathrm{D}) / \mathrm{I}(\mathrm{G})$ ratios $\sim 3$. We further note that some of these selected substrates already combine both features: $\mathrm{hBN}$ is not only flat but also hydrophobic ${ }^{16}$ and certain $\mathrm{SAMs}$ on $\mathrm{SiO}_{2}$ substrates are hydrophobic and can be flatter than $\mathrm{SiO}_{2}{ }^{15}$ The natural conclusion is that both the hydrophobicity and flatness of the substrate are prerequisites to obtain high-quality graphene. Both characteristics have a linked impact on the resulting doping and strain levels of graphene and determine the limits for measured optical and electronic properties. The economical and scalable flat and hydrophobic substrates for the handling and production of graphene layers are therefore of paramount importance for the fabrication of high performance graphene-based devices, regardless of scale.

Here, we demonstrate the use of a flat, hydrophobic polymeric layer as an alternative graphene exfoliation substrate to $\mathrm{SiO}_{2}$ in order to achieve high-quality flakes. We note that the exfoliation of graphene on polymer substrates has already been reported in the literature but without comparing the Raman spectral characteristics to the roughness of the polymer surface and the graphene quality. ${ }^{17-20}$

We use poly(methylmethacrylate) (PMMA) which is hydrophobic and can exhibit a surface roughness comparable to that observed for two dimensional materials supported by $\mathrm{SiO}_{2}{ }^{12,21}$ and smaller than the roughness of bare $\mathrm{SiO}_{2}$. Unless otherwise specified, a layer of hydrophilic polyvinyl acetate (PVA) is spun before spinning PMMA during preparation of the substrate. Graphene flakes exfoliated on these (PMMA/ PVA) layers consistently display large $\mathrm{I}(2 \mathrm{D}) / \mathrm{I}(\mathrm{G})$ ratios, 
approaching 10 in several cases, as well as showing other indicators of low residual doping and strain comparable to pristine, freestanding flakes. Importantly, similar Raman features are observed when varying the substrate thickness and composition by avoiding the usage of the PVA layer, and the aforementioned Raman features are also maintained when the same flakes are transferred to a different environment by encapsulation between hBN layers. Both these observations exclude the possibility that the high $\mathrm{I}(2 \mathrm{D}) / \mathrm{I}(\mathrm{G})$ ratios seen are an artifact due to optical interference effects arising from the specific values of the thickness of the selected (PMMA/PVA) dielectric layers. ${ }^{22}$ Finally, we demonstrate the consistent observation of carrier mobilities over $50000 \mathrm{~cm}^{2} \mathrm{~V}^{-1} \mathrm{~s}^{-1}$ in $\mathrm{hBN}$ encapsulated devices when using graphene originally exfoliated on a flat PMMA polymer. These mobilities are above the average of those reported for an ensemble ${ }^{23}$ of fully hBN encapsulated devices which used $\mathrm{SiO}_{2}$ as the initial exfoliation substrate $^{23,24}$ and make possible a more reproducible fabrication of high mobility encapsulated graphene van der Waals heterostructures.

The polymeric substrate is fabricated as follows: PVA ( $15 \%$ dissolved in deionized water) is spun at $2000 \mathrm{rpm}$ on $500 \mu \mathrm{m} \mathrm{Si} / 300 \mathrm{~nm} \mathrm{SiO}_{2}$ thermally oxidized substrates and baked out at $100^{\circ} \mathrm{C}$ for $2 \mathrm{~min}$. PMMA ( $4 \%$ dissolved in chlorobenzene) is spun on top at $2000 \mathrm{rpm}$ and baked at $180^{\circ} \mathrm{C}$ for $2 \mathrm{~min}$. We emphasize the critical importance of the polymer concentration, spin coating speed, and solvent in order to achieve flat PMMA films. ${ }^{25,26}$ Graphene is then mechanically exfoliated on top of PMMA and initially identified by optical contrast (supplementary material). An atomic force microscope (AFM, Bruker Innova, tapping mode) equipped with an AFM cantilever having a characteristic spring constant of $k=40 \mathrm{~N} / \mathrm{m}$ is used to measure the roughness of the flakes and substrates. ${ }^{7,12,27}$ Figure 1(a) shows an AFM image of a graphene flake on the PMMA surface. The measured surface roughness (given by the standard deviation of a fitted Gaussian) of graphene flakes exfoliated on top of this polymer on $1 \mu \mathrm{m}^{2}$ scan windows is $0.16 \pm 0.03 \mathrm{~nm}$ [Fig. 1(b)]. These values are similar to those observed for graphene exfoliated on hBN (roughness $\sim 0.1 \mathrm{~nm}$ ) ${ }^{12}$ and lower than those of graphene exfoliated on $\mathrm{SiO}_{2}[0.22 \pm 0.04 \mathrm{~nm}$, Fig. 1(b); in agreement with Ref. 12]. We note that the black dots in Fig. 1(a) are pinholes in the PMMA layer, which are commonly observed after spin-coating and baking this polymer. ${ }^{28,29}$ Graphene on these pinholes is locally suspended and indented during the AFM measurements since our tip is specifically selected to measure supported flakes. The presence of these pinholes, however, does not affect the conclusions of Fig. 1(b): pinholes increase the measured surface roughness of graphene flakes on PMMA as compared to regions between pinholes, but the overall roughness with pinholes included is still smaller than the roughness of graphene on $\mathrm{SiO}_{2}$.

Raman spectroscopy ${ }^{30}$ (Thermo Scientific DXR, $455 \mathrm{~nm}$ excitation, $50 \times$ objective, $\sim 1 \mu \mathrm{m}^{2}$ spot size) is used to assess the quality of more than 50 graphene flakes exfoliated on PMMA/PVA in terms of strain, doping, and defects in the graphene layers, ${ }^{11,27,30}$ by inspection of the peak intensity ratio, $\mathrm{I}(2 \mathrm{D}) / \mathrm{I}(\mathrm{G})$, as well as the full-width-at-half-maximum (FWHM) and position (Pos) variations of the G and 2D
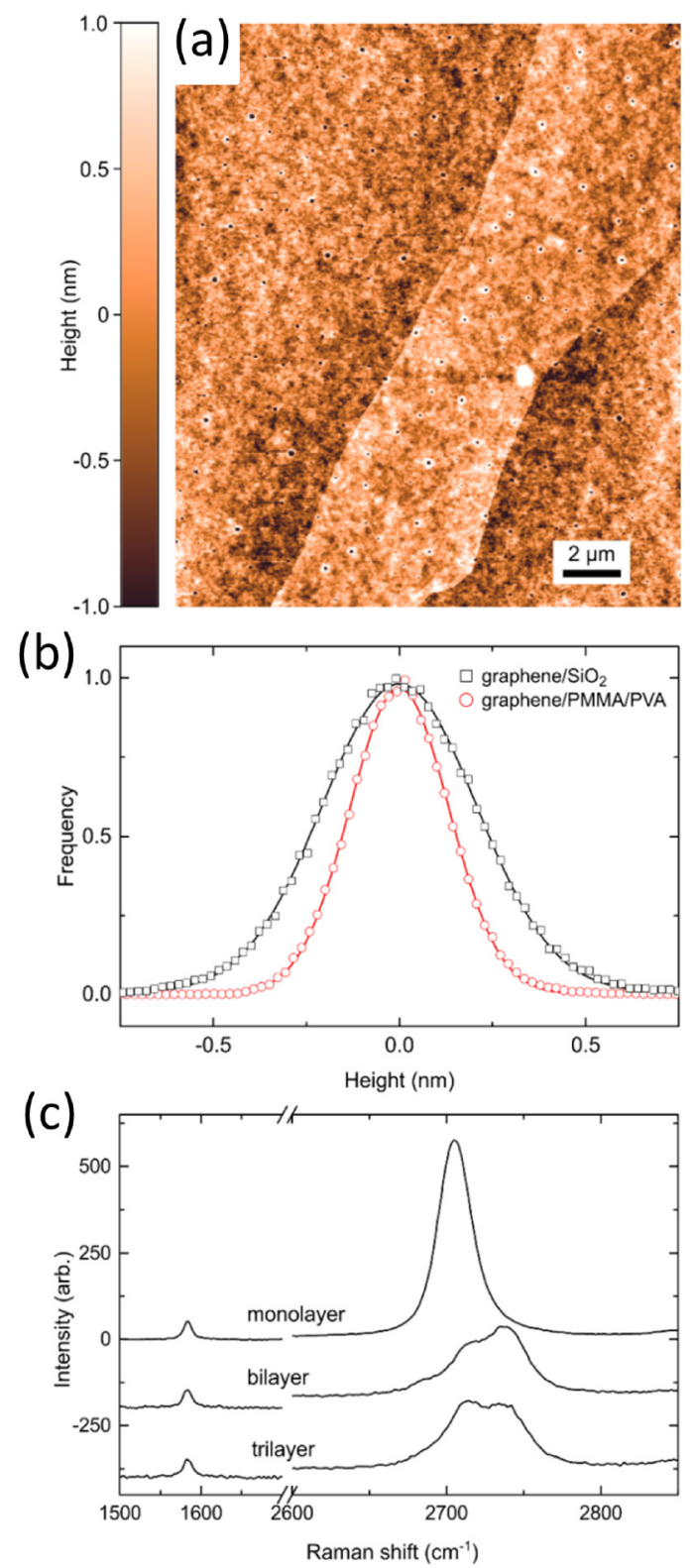

FIG. 1. Characterization of graphene flakes on PMMA/PVA. (a) AFM image of the monolayer graphene; the mapped area covers both a graphene flake and bare PMMA surface. The step size in the XY plane is $20 \mathrm{~nm}$. Black dots in the image are pinholes, features commonly appearing in spin-coated PMMA films ${ }^{28,29}$ where graphene is locally suspended. (b) Histogram of the height distribution (surface roughness) measured for graphene on $\mathrm{SiO}_{2}$ (black squares) and for graphene on PMMA/PVA (red circles) by AFM on $1 \mu \mathrm{m}^{2}$ scan windows. Solid lines are Gaussian fits to the distributions. (c) Raman spectra of mono-, bi,- and trilayer graphene normalized by the $G$ peak intensities. The spectra are collected on PMMA/PVAsupported flakes and show a higher $\mathrm{I}(2 \mathrm{D}) / \mathrm{I}(\mathrm{G})$ ratio as compared to usual values obtained on $\mathrm{SiO}_{2}$ supported graphene.

peaks. The Raman spectra were acquired with a power of 1 $\mathrm{mW}$ or lower in order to avoid the heating of graphene on the polymer substrate. The error in wavenumber determination in the Raman measurements is $+/-2 \mathrm{~cm}^{-1}$. Raman spectra of these graphene flakes on PMMA/PVA are shown in Fig. 1(c). For monolayer graphene, the observed I(2D)/ $\mathrm{I}(\mathrm{G})$ peak intensity ratio approaches 10 , with the position of the $\mathrm{G}$ peak, $\operatorname{Pos}(\mathrm{G}) \sim 1582 \mathrm{~cm}^{-1}$, and its full-width-at-halfmaximum, $\operatorname{FWHM}(\mathrm{G}) \sim 14 \mathrm{~cm}^{-1}$. All these features compare favorably to free-standing ${ }^{11}$ or fully encapsulated graphene, ${ }^{31}$ with very low implied levels of strain and 
doping. ${ }^{11,27,30,31}$ We also note the appearance of unusually large peak intensity ratios for bilayer and trilayer flakes [Fig. 1(c)] of $\mathrm{I}(2 \mathrm{D}) / \mathrm{I}(\mathrm{G})>3$ for these flakes on PMMA/PVA: peak ratios on $\mathrm{SiO}_{2}$ are typically below $1 .{ }^{27,30,31}$

We categorize more than 50 measured monolayer graphene flakes exfoliated on PMMA/PVA according to their average $\mathrm{I}(2 \mathrm{D}) / \mathrm{I}(\mathrm{G})$ ratios, [Fig. 2(a)]: $\mathrm{I}(2 \mathrm{D}) / \mathrm{I}(\mathrm{G})<4$ (white dots), $4<\mathrm{I}(2 \mathrm{D}) / \mathrm{I}(\mathrm{G})<8$ (grey dots), and $\mathrm{I}(2 \mathrm{D}) / \mathrm{I}(\mathrm{G})>8$ (black dots). More than $60 \%$ of the studied graphene flakes have a $\mathrm{I}(2 \mathrm{D}) / \mathrm{I}(\mathrm{G})$ ratio $>4$ and more than $30 \%$ have ratios $\mathrm{I}(2 \mathrm{D}) / \mathrm{I}(\mathrm{G})>8$. By comparison, graphene on $\mathrm{SiO}_{2}$ only shows $\mathrm{I}(2 \mathrm{D}) / \mathrm{I}(\mathrm{G})<2^{27,30}$ and $\mathrm{I}(2 \mathrm{D}) / \mathrm{I}(\mathrm{G})<4$ on hydrophobized $\mathrm{SiO}_{2}{ }^{32}$ and on $\mathrm{hBN}^{31}$ Figure 2(a) shows the correlation between the 2D and $G$ peak positions of these flakes [Pos(2D) vs $\operatorname{Pos}(\mathrm{G})$ ], commonly used to separate and obtain specific information about strain and doping levels existent in nondefective graphene. ${ }^{33-35}$ Dashed lines in the figure represent the predicted evolution of the Raman parameters in the unique presence of one of the mechanisms, i.e., strain (grey dotted line) or doping (black dashed line). These lines intersect (asterisk) at $\operatorname{Pos}(\mathrm{G})^{0}=1582 \mathrm{~cm}^{-1}$ and $\operatorname{Pos}(2 \mathrm{D})^{0}=2700 \mathrm{~cm}^{-1}$, which correspond to pristine (strain-free, doping-free) graphene flakes. ${ }^{36}$ We note that while the value of the $G$ peak position $\operatorname{Pos}(\mathrm{G})^{0}=1582 \mathrm{~cm}^{-1}$ is well-established for clean, freestanding
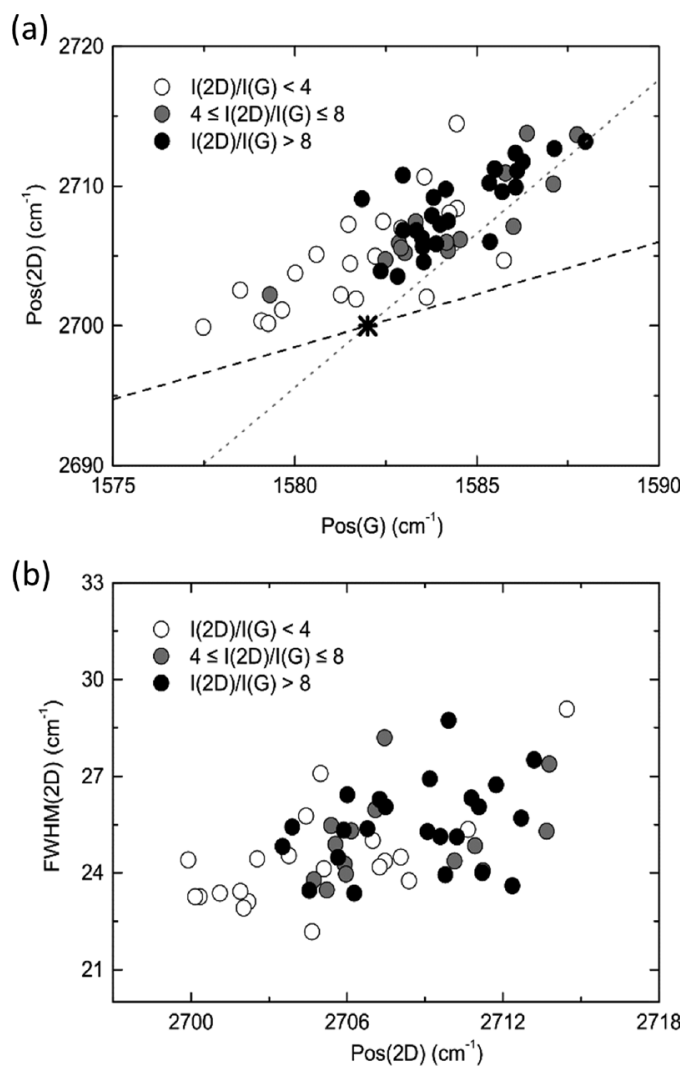

FIG. 2. Raman analysis of PMMA-supported single layer graphene. Data points are categorized by $\mathrm{I}(2 \mathrm{D}) / \mathrm{I}(\mathrm{G})$ as indicated. (a) Correlation between the $\mathrm{G}$ and 2D Raman peaks frequencies. Data points are from single point spectra of $>50$ different single layer flakes. The predicted evolution ${ }^{33-35}$ in the unique presence of strain (grey dotted line) or doping (black dashed line) is also plotted and intersects at a point (asterisk) representing doping- and strain-free graphene $\left[\operatorname{Pos}(G)^{0}, \operatorname{Pos}(2 D)^{0}\right]$. Dispersion of the data around this intersection is very small - with $\Delta \operatorname{Pos}(\mathrm{G})<5 \mathrm{~cm}^{-1}$ and $\Delta \operatorname{Pos}(2 \mathrm{D})<13 \mathrm{~cm}^{-1}$ showing both a low level of doping and strain in the exfoliated flakes and low variation in these quantities. (b) Correlation between Pos(2D) and FWHM(2D) for the PMMA-supported exfoliated single layer graphene flakes. or encapsulated graphene, ${ }^{11,21}$ the exact position of the $2 \mathrm{D}$ peak is subject to discussion. ${ }^{36-38}$ Small deviations in $\operatorname{Pos}(2 \mathrm{D})^{0}$ occur due to the subtle interplay between the phononic and electronic energy dispersions,${ }^{37}$ which ultimately depend on the surrounding environment. ${ }^{36,38}$ For our purposes, we adopt the calculated $\operatorname{Pos}(2 \mathrm{D})^{0}=2700 \mathrm{~cm}^{-1}$ as the value in pristine graphene for our laser excitation energy $(2.72 \mathrm{eV}) .^{36,38}$ With these considerations, we can confirm the small variations between the $G$ peak positions $\Delta \operatorname{Pos}(G)=\left(\operatorname{Pos}(G)-\operatorname{Pos}(G)^{0}\right) \leq 5 \mathrm{~cm}^{-1}$ and $2 \mathrm{D}$ peak positions $\Delta \operatorname{Pos}(2 \mathrm{D})=\left(\operatorname{Pos}(2 \mathrm{D})-\operatorname{Pos}(2 \mathrm{D})^{0}\right) \leq 13 \mathrm{~cm}^{-1}$ for all the flakes under study with respect to the pristine case $\left[\operatorname{Pos}(G)^{0}, \operatorname{Pos}(2 \mathrm{D})^{0}\right]$. Furthermore, flakes on PMMA/PVA show a full-width-at-half-maximum of the 2D peak [FWHM(2D)] lying between 22 and $28 \mathrm{~cm}^{-1}$ [Fig. 2(b)], also similar to pristine, freestanding graphene. ${ }^{11}$ Quantitative values of strain and doping can be calculated from these Raman signals. ${ }^{33-35}$ Specifically, in our flakes with ratios $\mathrm{I}(2 \mathrm{D}) / \mathrm{I}(\mathrm{G})>8$, we extract averaged strain levels of $-0.17 \%$ and doping levels below $2.5 \times 10^{12} \mathrm{~cm}^{-1}$ (supplementary material). These values are well below strain and doping values estimated for graphene on $\mathrm{SiO}_{2},{ }^{27,33-35}$ typically larger than $0.2 \%$ and $5 \times 10^{12} \mathrm{~cm}^{-1}$ for strain and doping, respectively, in accordance with the expectations from the higher $\mathrm{I}(2 \mathrm{D}) / \mathrm{I}(\mathrm{G})$ ratio of these flakes.

Thus, our combined AFM and Raman data indicate that dry and flat PMMA itself is harmless to graphene. Instead, the associated usage of solvents, ${ }^{19,20}$ heat treatments, ${ }^{6,7}$ and/or substrate roughness ${ }^{7,21}$ are responsible for the degradation of the optical and electronic properties of this two-dimensional material.

Next, to eliminate the possibility that the high $\mathrm{I}(2 \mathrm{D}) / \mathrm{I}(\mathrm{G})$ ratios appearing on PMMA/PVA arise incidentally from interference effects caused by the selected dielectric layers, ${ }^{22}$ we have confirmed that large Raman $\mathrm{I}(2 \mathrm{D}) / \mathrm{I}(\mathrm{G})$ ratios are also observed when changing the composition and thickness of the substrate by eliminating the PVA layer (supplementary material), i.e., when graphene is supported purely by the $\sim 200 \mathrm{~nm}$ thick PMMA layer. We have additionally measured the Raman characteristics of selected graphene flakes before and after encapsulation with $\mathrm{hBN}^{23,24}$ and have confirmed that the high $\mathrm{I}(2 \mathrm{D}) / \mathrm{I}(\mathrm{G})$ ratios are conserved after this complete change of the environment of the graphene. For this task, commercially available hexagonal boron nitride crystals (HQ Graphene) are exfoliated on oxidized silicon substrates (300 $\mathrm{nm}$ of $\mathrm{SiO}_{2}$ thermally grown on top of highly doped silicon) and are used as encapsulation for heterostructure devices. In assembling heterostructures, we aim to maintain the low levels of strain and doping observed in the graphene on PMMA samples. We largely follow the assembly methods presented in Ref. 23 using polypropylene carbonate (PPC) as a transfer polymer to pick up and drop down hBN and graphene but with some modifications [Figs. 3(a)-(c)].

We observe by AFM that exposing graphene exfoliated on PMMA to temperatures above $70^{\circ} \mathrm{C}$ has a detrimental effect on the roughness, with the surface roughness increasing above $0.4 \mathrm{~nm}$ at $100^{\circ} \mathrm{C}$. Such temperatures are unnecessary in the assembly presented here however, as the graphene flakes are already clean and dry on the hydrophobic PMMA. By performing assembly at $60^{\circ} \mathrm{C}$, just over the glass transition temperature of the PPC, the drop down of an hBN flake on top of graphene can be accomplished without increasing the 
(a)

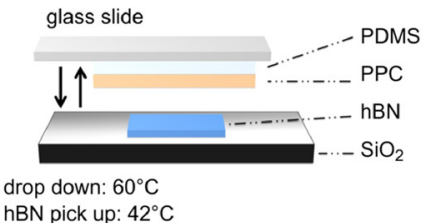

(b)

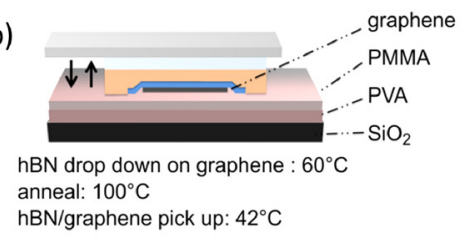

(c)

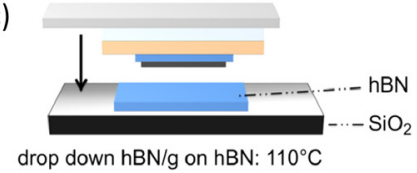

(d)
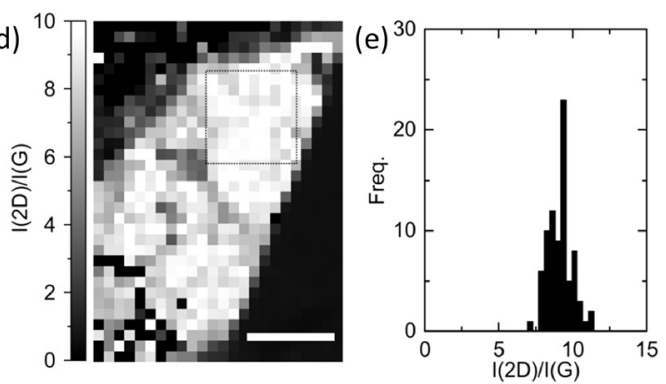

(f)

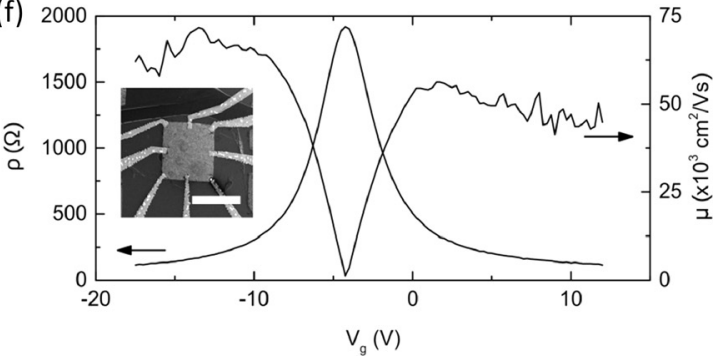

FIG. 3. Stacking method and characterization of encapsulated graphene flakes. (a)-(c) Schematic illustration of the developed stacking procedure, which partially follows the method published in Ref. 23, used to realize hBN/graphene/hBN van der Waals heterostructures. (d) Raman map showing the $\mathrm{I}(2 \mathrm{D}) / \mathrm{I}(\mathrm{G})$ ratio of a representative encapsulated graphene flake. The scale bar is $5 \mu \mathrm{m}$. (e) Histogram of $\mathrm{I}(2 \mathrm{D}) /$ $\mathrm{I}(\mathrm{G})$ ratios for the squared indicated region of $5 \mu \mathrm{m}$ side in (d). (f) Resistivity and mobility of the device. Inset: SEM micrograph of the measured device. The scale bar is $5 \mu \mathrm{m}$. roughness. After the hBN flake has been fully dropped down on top of graphene [Fig. 3(b)], the partial heterostructure can safely be exposed to a temperature ramp from $60{ }^{\circ} \mathrm{C}$ to $100^{\circ} \mathrm{C}$ over $5 \mathrm{~min}$ to ensure adhesion between the layers. The two flakes are then lifted together and dropped down on top of the bottom hBN [Fig. 3(c)] at $110^{\circ} \mathrm{C}$, similar to Ref. 23 , to avoid strain in the final heterostructure. We note that it is possible to perform this last "drop-down" step at $110^{\circ} \mathrm{C}$ since graphene supported by $\mathrm{hBN}$ will not corrugate at this temperature. Raman maps demonstrate the lack of strain and doping introduced to the graphene by this modified encapsulation process when using pristine, preselected graphene flakes on PMMA/ PVA substrates. In particular, Fig. 3(d) shows a homogeneous encapsulated flake with $\mathrm{I}(2 \mathrm{D}) / \mathrm{I}(\mathrm{G})$ ratios higher than 8 and $\operatorname{Pos}(\mathrm{G})$ around $1582 \mathrm{~cm}^{-1}$, similar to the ratio of the graphene flake on PMMA/PVA prior to its encapsulation (supplementary material). Additional data on the evolution of the Raman features of graphene from polymer substrates before and after encapsulation with $\mathrm{hBN}$ are given in the supplementary material.

Subsequently to the fabrication of encapsulated samples and the measurement of the Raman spectral characteristics before and after this process, we fabricate electrical devices for measurement of the corresponding carrier mobility values. Although it would be highly desirable to verify that graphene flakes on flat PMMA have a high mobility, i.e., without resorting to additional $\mathrm{hBN}$ encapsulation steps, we note that this is not trivial since PMMA is not compatible with standard electron beam lithography based fabrication steps typically used to contact graphene. Alternative contacting methods such as shadow masks ${ }^{7}$ or lithography-free microprobing techniques ${ }^{39}$ are difficult to apply here due to reduced flake sizes obtained (most of them around $10 \mu \mathrm{m} \times 10 \mu \mathrm{m}$, see supplementary material). State-of-the-art nanoprobing techniques ${ }^{40}$ could be a possible way to measure the mobility of these flakes.

Based on the assumption that the mobility values obtained for a graphene flake are correlated with the Raman spectral properties and in order to give an indicative mobility value that might be expected from the polymer supported flakes here described, we have measured the mobility of hBN encapsulated devices produced from PMMA exfoliated and preselected high $\mathrm{I}(2 \mathrm{D}) / \mathrm{I}(\mathrm{G})$ ratio graphene flakes. To undertake these measurements, we define $5 \mu \mathrm{m} \times 5 \mu \mathrm{m}$ squareshaped regions and add electrical contacts using standard fabrication techniques. ${ }^{23,24}$ The homogeneity of the samples was first investigated by comparing the resistance $R$ in two perpendicular configurations (see supplementary material). Figure 3(f) shows the room temperature resistivity $\rho$ and field-effect mobility $\mu$ as a function of the back-gate voltage $V_{\mathrm{g}}$ for one of the fabricated devices. These parameters are calculated from $\rho=\frac{\pi}{\ln 2} R$ (resistivity calculation with the van der Pauw configuration for square geometries ${ }^{24}$ ) and $\mu=\frac{t}{\varepsilon} \frac{\partial \sigma}{\partial V_{g}}$, respectively, where $\varepsilon$ is the dielectric permittivity, $t$ is the dielectric thickness $\left(300 \mathrm{~nm} \mathrm{SiO}_{2}, 10 \mathrm{~nm} \mathrm{hBN}\right)$, and $\sigma=1 / \rho$ is the electrical conductivity of the device. Carrier mobilities above $50000 \mathrm{~cm}^{2} \mathrm{~V}^{-1} \mathrm{~s}^{-1}$ were measured for both electron and holes [Fig. 3(f)] in all the examined devices at room temperature.

In conclusion, we have shown that exfoliating graphene on a hydrophobic, flat polymeric substrate leads to very low residual strain, doping, and roughness as compared to the same operation on $\mathrm{SiO}_{2}$. Whilst such flakes hold promise for electronic and optical studies - particularly those where a transparent and flexible substrate is required-without substantial further processing, we have additionally shown that these graphene flakes are ideally suited for the reproducible fabrication of encapsulated van der Waals heterostructures with mobilities above $50000 \mathrm{~cm}^{2} \mathrm{~V}^{-1} \mathrm{~s}^{-1}$. We anticipate that the scheme presented for reducing strain and doping in graphene is applicable to other two-dimensional materials, which in general will enable the fabrication of related heterostructures with properties that more consistently approach theoretical limits. From a practical perspective, Raman spectroscopy is ideal for rapidly prescreening graphene flakes, suggesting that such an approach can be integrated $^{41}$ into automated systems for the assembly of van der Waals heterostructures in order to avoid producing heterostructures with non-idealities. Furthermore, the ability to exfoliate graphene on flat PMMA not only reduces the effects of strain and doping on the graphene but also provides for a much reduced cost of substrate with arbitrary size as compared to oxidized silicon. 
See supplementary material containing an optical image of graphene on PMMA/PVA, the evolution of Raman spectra of graphene flakes on PMMA/PVA and after hBN encapsulation and electrical measurements verifying the homogeneity of hBN encapsulated graphene devices, quantitative estimation of strain and doping levels on graphene flakes on PMMA/PVA, Raman spectra of graphene on PMMA/SiO and surface roughness of spin-coated PVA films.

We acknowledge stimulating discussions with L. Camilli. This work was supported by the Danish National Research Foundation Center for Nanostructured Graphene, Project No. DNRF103, the EU Seventh Framework Programme (FP7/ 2007-2013) under Grant Agreement No. FP7-6040007 "GLADIATOR," and the EU H2020 Graphene Flagship Core 1, Grant Agreement No. 696656.

${ }^{1}$ Y. Huang, E. Sutter, N. N. Shi, J. Zheng, T. Yang, D. Englund, H.-J. Gao, and P. Sutter, ACS Nano 9(11), 10612 (2015).

${ }^{2}$ B. Luo, J. M. Caridad, P. R. Whelan, J. D. Thomsen, D. M. A. Mackenzie, A. G. Cabo, S. K. Mahatha, M. Bianchi, P. Hofmann, P. U. Jepsen, P. Bøggild, and T. J. Booth, 2D Mater. 4, 045017 (2017).

${ }^{3}$ P. Blake, E. W. Hill, A. H. Castro Neto, K. S. Novoselov, D. Jiang, R. Yang, T. J. Booth, and A. K. Geim, Appl. Phys. Lett. 91, 063124 (2007).

${ }^{4}$ K. S. Novoselov, A. K. Geim, S. V. Morozov, D. Jiang, Y. Zhang, S. V. Dubonos, I. V. Grigorieva, and A. A. Firsov, Science 306, 666 (2004).

${ }^{5}$ Y. Zhang, Y. Tan, H. L. Stormer, and P. Kim, Nature 438, 201 (2005).

${ }^{6}$ S. Ryu, L. Liu, S. Berciaud, Y. Yu, H. Liu, P. Kim, G. W. Flynn, and L. E. Brus, Nano Lett. 10, 4944 (2010).

${ }^{7}$ L. Gammelgaard, J. M. Caridad, A. Cagliani, D. M. A. Mackenzie, D. H. Petersen, T. J. Booth, and P. Bøggild, 2D Mater. 1, 035005 (2014).

${ }^{8}$ J. Chen, C. Jang, S. Xiao, M. Ishigami, and M. S. Fuhrer, Nat. Nanotechnol. 3, 206 (2008).

${ }^{9}$ E. H. Hwang and S. Das Sarma, Phys. Rev. B 77, 115449 (2008).

${ }^{10}$ J. H. Chen, C. Jang, S. Adam, M. S. Fuhrer, E. D. Williams, and M. Ishigami, Nat. Phys. 4, 377 (2008).

${ }^{11}$ S. Berciaud, S. Ryu, L. E. Brus, and T. F. Heinz, Nano Lett. 9, 346 (2009).

${ }^{12}$ C. R. Dean, A. F. Young, I. Meric, C. Lee, L. Wang, S. Sorgenfrei, K. Watanabe, T. Taniguchi, P. Kim, K. L. Shepard, and J. Hone, Nat. Nanotechnol. 5, 722 (2010).

${ }^{13}$ M. Lafkioti, B. Krauss, T. Lohmann, U. Zschieschang, H. Klauk, K. V. Klitzing, and J. H. Smet, Nano Lett. 10, 1149 (2010).

${ }^{14}$ J. M. Caridad, S. Connaughton, C. Ott, H. B. Weber, and V. Krstić, Nat. Commun. 7, 12894 (2016).

${ }^{15}$ S.-Y. Chen, P.-H. Ho, R.-J. Shiue, C.-W. Chen, and W.-H. Wang, Nano Lett. 12, 964 (2012).

${ }^{16}$ S. M. Kim, A. Hsu, M. H. Park, S. H. Chae, S. J. Yun, J. S. Lee, D.-H. Cho, W. Fang, C. Lee, T. Palacios, M. Dresselhaus, K. K. Kim, Y. H. Lee, and J. Kong, Nat. Commun. 6, 8662 (2015).
${ }^{17}$ F. Traversi, F. J. Guzman-Vázquez, L. G. Rizzi, V. Russo, C. S. Casari, C. Gómez-Navarro, and R. Sordan, New J. Phys. 12, 023034 (2010).

${ }^{18}$ H. M. Wang, Z. Zheng, Y. Y. Wang, J. J. Qiu, Z. B. Guo, Z. X. Shen, and T. Yu, Appl. Phys. Lett. 96, 023106 (2010).

${ }^{19}$ C. R. Dean, A. F. Young, P. Cadden-Zimansky, L. Wang, H. Ren, K. Watanabe, T. Taniguchi, P. Kim, J. Hone, and K. L. Shepard, Nat. Phys. 7, 693 (2011).

${ }^{20}$ A. S. Mayorov, R. V. Gorbachev, S. V. Morozov, L. Britnell, R. Jalil, L. A. Ponomarenko, P. Blake, K. S. Novoselov, K. Watanabe, T. Taniguchi, and A. K. Geim, Nano Lett. 11, 2396 (2011).

${ }^{21}$ L. Banszerus, H. Janssen, M. Otto, A. Epping, T. Taniguchi, K. Watanabe, B. Beschoten, D. Neumaier, and C. Stampfer, 2D Mater. 4, 025030 (2017).

${ }^{22}$ D. Yoon, H. Moon, Y.-W. Son, J.-S. Choi, B.-H. Park, Y.-H. Cha, Y.-D. Kim, and H. Cheong, Phys. Rev. B 80, 125422 (2009).

${ }^{23}$ F. Pizzocchero, L. Gammelgaard, B. S. Jessen, J. M. Caridad, L. Wang, J. Hone, P. Bøggild, and T. J. Booth, Nat. Commun. 7, 11894 (2016).

${ }^{24}$ L. Wang, I. Meric, P. Y. Huang, Q. Gao, Y. Gao, H. Tran, T. Taniguchi, K. Watanabe, L. M. Campos, D. A. Muller, J. Guo, P. Kim, J. Hone, K. L. Shepard, and C. R. Dean, Science 342, 614 (2013).

${ }^{25}$ N. C. H. Le, V. Gubala, E. Clancy, T. Barry, T. J. Smith, and D. E. Williams, Biosens. Bioelectron. 36, 250 (2012).

${ }^{26}$ X. Zhang, K. G. Yager, S. Kang, N. J. Fredin, B. Akgun, S. Satija, J. F. Douglas, A. Karim, and R. L. Jones, Macromolecules 43, 1117 (2010).

${ }^{27}$ J. M. Caridad, F. Rossella, V. Bellani, M. Maicas, M. Patrini, and E. Díez, J. Appl. Phys. 108, 084321 (2010).

${ }^{28}$ N. G. Semaltianos, Microelectron. J. 38, 754 (2007).

${ }^{29}$ T. Tippo, C. Thanachayanont, P. Muthitamongkol, C. Junin, M. Hietschold, and A. Thanachayanont, Thin Solid Films 546, 180-184 (2013).

${ }^{30}$ A. C. Ferrari, J. C. Meyer, V. Scardaci, C. Casiraghi, M. Lazzeri, F. Mauri, S. Piscanec, D. Jiang, K. S. Novoselov, S. Roth, and A. K. Geim, Phys. Rev. Lett. 97, 187401 (2006).

${ }^{31}$ L. Wang, Z. Chen, C. R. Dean, T. Taniguchi, K. Watanabe, L. E. Brus, and J. Hone, ACS Nano 6, 9314 (2012).

${ }^{32}$ X. Fan, R. Nouchi, and K. Tanigaki, J. Phys. Chem. C 115, 12960 (2011).

${ }^{33}$ J. Lee, G. Ahn, J. Shim, Y. S. Lee, and S. Ryu, Nat. Commun. 3, 1024 (2012).

${ }^{34}$ G. Ahn, H. R. Kim, T. Y. Ko, K. Choi, K. Watanabe, T. Taniguchi, B. H. Hong, and S. Ryu, ACS Nano 7, 1533 (2013).

${ }^{35}$ T. G. A. Verhagen, K. Drogowska, M. Kalbac, and J. Vejpravova, Phys. Rev. B 92, 125437 (2015).

${ }^{36}$ P. Venezuela, M. Lazzeri, and F. Mauri, Phys. Rev. B 84, 035433 (2011).

${ }^{37}$ F. Forster, A. Molina-Sanchez, S. Engels, A. Epping, K. Watanabe, T. Taniguchi, L. Wirtz, and C. Stampfer, Phys. Rev. B 88, 085419 (2013).

${ }^{38}$ R. Narula and S. Reich, Phys. Rev. B 78, 165422 (2008).

${ }^{39}$ P. Gant, Y. Niu, S. A. Svatek, N. Agrait, C. Munuera, M. GarcíaHernández, R. Frisneda, D. Perez de Lara, and A. Castellanos Gómez, J. Mater. Chem. C 5, 11252 (2017).

${ }^{40}$ E. Paul, H. Herzog, S. Jansen, C. Hobert, and E. Langer, Microelectron. Reliab. 54, 2115 (2014).

${ }^{41}$ J. M. Caridad, F. Rossella, V. Bellani, M. S. Grandi, and E. Diez, J. Raman Spectrosc. 42, 286 (2011). 